\title{
Structural proteomics-driven targeted design of favipiravir- binding site in the RdRp of SARS-CoV-2 unravels susceptible hotspots and resistance mutations
}

Aditya K. Padhi ${ }^{1}$, Jagneshwar Dandapat ${ }^{2,3}$, Vladimir N. Uversky ${ }^{4}$, and Timir Tripathi ${ }^{*}$

${ }^{1}$ Laboratory for Structural Bioinformatics, Center for Biosystems Dynamics Research, RIKEN, Yokohama, Kanagawa 230-0045, Japan

${ }^{2}$ Centre of Excellence in Integrated Omics and Computational Biology, Utkal University, Bhubaneswar 751004, Odisha, India

${ }^{3}$ Post Graduate Department of Biotechnology, Utkal University, Bhubaneswar 751004, Odisha, India

${ }^{4}$ Department of Molecular Medicine and Byrd Alzheimer's Research Institute, Morsani College of Medicine, University of South Florida, Tampa, FL 33620, United States

${ }^{5}$ Molecular and Structural Biophysics Laboratory, Department of Biochemistry, NorthEastern Hill University, Shillong- 793022, India

\section{${ }^{*}$ Corresponding author:}

Dr. Timir Tripathi, Department of Biochemistry, North-Eastern Hill University, Shillong793022, India. Email: timir.tripathi@gmail.com, Tel: +91-364-2722141; Fax: +91-3642550108 


\section{Abstract}

Favipiravir is a broad-spectrum inhibitor of viral RNA-dependent RNA polymerase (RdRp) currently being used to manage COVID-19 in several countries. By acting as a substrate for RdRp, favipiravir gets incorporated into the nascent viral RNA and prevents strand extension. A high mutation rate of SARS-CoV-2 RdRp may facilitate antigenic drift as an answer to the host immune response, thereby generating resistance of virus to favipiravir. Therefore, it is extremely crucial to predict potential mutational sites in the RdRp and the emergence of structural modifications contributing to drug resistance. Here, we used high-throughput interface-based protein design to generate $>100,000$ designs and identify mutation hotspot residues in the favipiravir-binding site of RdRp. Several mutants had lower binding affinities to favipiravir, out of which hotspot residues with a high propensity to undergo positive selection were identified. The results showed that the designs retained an average of 97 to $98 \%$ sequence identity, suggesting that SARS-CoV2 can develop favipiravir resistance with just a few mutations. Notably, we observed that out of 134 mutations predicted designs, 63 specific mutations were already present in the CoV-GLUE database, thus attaining $\sim 47 \%$ correlation match with the clinical sequencing data. The findings improve our understanding of the potential signatures of adaptation in SARS-CoV-2 against favipiravir and management of COVID-19. Furthermore, they can help develop exhaustive strategies for robust antiviral design and discovery.

\section{KEYWORDS}

Drug resistance; nsp12; protein design; fitness; RNA-dependent RNA polymerase; resistance mutations; SARS-CoV-2.

\section{RUNNING TITLE}

Favipiravir resistance sites in SARS-CoV-2 


\section{Introduction}

The SARS-CoV-2 RNA-dependent RNA polymerase (RdRp or nsp12-nsp7-nsp8 complex) has emerged as a critical drug target for treating COVID-19. It is an ideal target because it is the central enzyme involved in the virus replication and transcription, and its enzymatic counterpart is absent in host cells. The recent detailed characterization of structural and biochemical properties of SARS-CoV-2 RdRp has allowed the design and development of specific RdRp inhibitors [1, 2]. The major drug approved for treating hospitalized patients with COVID-19 is the RdRp inhibitor remdesivir, which received emergency use authorization (EUA) in several countries [3]. RdRps are highly conserved enzymes across different coronaviruses, with approximately $96 \%$ sequence identity between SARS-CoV and SARS-CoV-2 [4]. All coronavirus RdRps have conserved threedimensional (3D) right-handed structure, being organized into palm, thumb, and finger subdomains, as well as show conserved active site motifs. These features can be used to develop broad-spectrum inhibitors for these and other RNA viruses.

Favipiravir (6-fluoro-3-oxo-3,4-dihydropyrazine-2-carboxamide) is a broadspectrum drug originally developed to act against the influenza virus. In addition, it is effective against a wide variety of RNA viruses, including flaviviruses, hantaviruses, arenaviruses, phleboviruses, noroviruses, enteroviruses, yellow fever virus, foot-andmouth disease virus, Western equine encephalitis virus, Ebola virus, Lassa virus, rabies, respiratory syncytial virus, and rhinoviruses [5, 6]. For COVID-19, oral therapy with favipiravir has been recommended in several countries, such as Russia, India, Japan, Saudi Arabia, Thailand, and Kenya, and has been proven useful in managing mild to moderate cases of COVID-19 [7-9]. Furthermore, more than 40 clinical trials of favipiravir are in progress in various countries, including Japan, Italy, UK, USA, Canada, France, Germany, India, Thailand, Russia, Egypt, and Saudi Arabia, to treat patients with COVID19 (https://clinicaltrials.gov). Favipiravir treatment could rapidly clear viral load and induce faster clinical improvement. In India currently, it is a major antiviral presently used for the clinical management and treatment of COVID-19 [10, 11]. Favipiravir (prodrug) is a nucleoside analog that is metabolically activated via ribosylation and phosphorylation and is converted into favipiravir ribofuranosyl-5B-triphosphate (favipiravir-RTP). It is 
incorporated into the nascent viral RNA by viral RdRp resulting in chain termination or accumulation of deleterious mutations [12-14]. Recently, a cryo-EM structure of the SARS-CoV-2 RdRp in complex with favipiravir-RTP and template primer doublestranded RNA has been determined (PDB ID: 7AAP)[15]. Favipiravir binds to the active site of the viral RdRp, being mistaken as a purine nucleotide, transforming the binding pocket into a catalytically non-productive conformation. It stacks onto the 3 ' nucleotide of the primer strand and forms a non-canonical base pair with the template RNA strand using its amide group (Figure 1a-1c). Therefore, favipiravir functions as a chain terminator, inhibiting viral $R d R p$ by the termination of chain elongation at the site of incorporation.

Since its emergence in December 2019, SARS-CoV-2 has undergone more than 50,000 mutations and recombination events, as compared to the Wuhan reference genome (NC_045512.2) (http://cov-glue.cvr.gla.ac.uk/\#/home). Until December 2020, at least 198 sites in the viral genome have already undergone recurrent, independent mutations [16]. Although the rate is lower than that of influenza and human immunodeficiency virus (HIV), SARS-CoV-2 is presently accumulating at least two mutations per month in its genome [17]. The continuous emerging mutations are a cause of concern, as they could hinder the development of long-lasting and effective therapeutics. These concerns are supported by the observations of the natural favipiravir resistance emergence in Chikungunya virus [18] and enterovirus 71 [19], and development of the favipiravir resistance by the pandemic H1N1 influenza $A$ virus in the laboratory studies [20]. Chances of the emergence of favipiravir resistant SARS-CoV-2 strains increase due to its intensive use as the COVID-19 pandemic progresses. We recently identified potential residues that could contribute to remdesivir and molnupiravir resistance in SARS-CoV-2 [21], as well as identified potential signatures of SARS-CoV-2 main protease adaptation against boceprevir and telapravir[22]. We believe the knowledge of potential mutants is essential and can help design new and more effective drugs and guide the clinicians to properly manage and administer the treatment regimen. In this study, we used a high-throughput interface-design strategy to predict potential mutation hotspots and resistance sites in the favipiravir binding site in the RdRp of SARSCoV-2. 


\section{Materials and methods}

\section{Identification favipiravir and nsp12 interacting residues}

The cryo-EM structure of SARS-CoV-2 RdRp (nsp7-nsp8-nsp12) in complex with the template:primer dsRNA and favipiravir-RTP (PDB code: 7AAP) was used to determine the interactions between nsp12 and favipiravir. This analysis resulted in 72 nsp12interacting residues with favipiravir. The complex structure was subsequently processed using the Molecular Operating Environment (MOE 2018.01, v2021.03).

\section{MOE-based resistance scan design methodology}

To identify potential single point mutations of nsp12 that could develop resistance against favipiravir, MOE's resistance scan methodology was used (MOE 2018.01, v2021.03) [23]. The prepared complex structure was used as an input, and 41 nsp12 residues that interacted with favipiravir were designed with naturally sampled SNPs, whereas the remaining residues that interacted with RNA were not selected for the design. During the design, the template:primer dsRNA was retained in the complex structure. The rotamer explorer option of the ensemble protein design protocol was enabled due to the flexibility of the designed site. As discussed in our recent report[22], various physico-chemical parameters were set accordingly, and a total of 350 designs were generated. Parameters such as affinity and stability of the designed complexes were examined. The relative binding affinity of the mutation to the wild-type protein (dAffinity) was computed as described in our recent report[22]. This methodology served as an indirect computational fitness test model, sampling only those mutations that were not deleterious and were more likely to develop naturally.

\section{Rosetta-based structural refinement and ligand-based interface design}

The complex structure prepared using MOE was next used for Rosetta-based design [2427]. First, parameters for favipiravir compatible with Rosetta forcefield were generated by 
adding appropriate charges and following Rosetta's appropriate parameter generation protocol. Second, the complex was energy minimized using Rosetta relax [25]. Five models were generated, and the lowest energy complex structure of the favipiravir-bound complex was used for the targeted interface design experiment. The Rosetta macromolecular modeling suite was used to redesign nsp12 with backbone flexibility [24]. In this step, 41 residues of nsp12 that constituted the favipiravir-interacting site were designed with the naturally sampled SNPs, whereas the remaining residues that interact with RNA and all other residues of nsp7, nsp8, and nsp12 were allowed to only repack without design. In the design experiments, a modified RosettaScript was used to sample the favipiravir-interacting residues of nsp12, which considered minor movements of the backbone of nsp12 to prevent steric clashes with ligands after introducing mutations [26]. The Rosetta all-atom forcefield was used to sample the designs. A total of 100,000 designs of favipiravir-bound complexes were generated, and the Rosetta total score, RMSD from the initial structure, Rosetta interface delta (denoting the binding affinities between the designed nsp12 and favipiravir), and the percent sequence identities from the initial nsp12 sequence were analyzed to understand the mutational landscapes and effect of mutations on the physicochemical characteristics of nsp12 and the overall complex.

\section{Validation of the design protocol}

The accuracy and predicting ability of our MOE-based and Rosetta-based interface design protocol were validated on a well-studied remdesivir-resistant mutant of nsp12, named V557L of SARS-CoV [28]. As described in our recent study[22], first, the MOE resistance scan methodology was used, where Val557 was designed with $A, D, E, G, I$, $L, M$, and $F$, and the affinities between designed nsp12 and remdesivir were calculated. Second, the Rosetta interface design protocol was used, where Val557 was designed with naturally sampled SNP residues, although the remaining nsp12 residues were only repacked without design. In this run, 5000 designs were generated and analyzed for the designed mutations with their respective total scores. Finally, the resistance mutations from our design calculations were compared with the known SARS-CoV-2 nsp12 
sequences available in the CoV-GLUE database [29]. The frequency of the mutations retrieved from CoV-GLUE was compared with the favipiravir binding site of nsp12 to determine the accuracy of our design methodology.

\section{Mutational landscapes of the top-ranked affinity-attenuating and affinity-enhancing designs}

WebLogo was used to obtain and plot the type and frequency of designed amino acid residues in the 41 favipiravir-interacting residues of nsp12 from the top-ranked affinityenhanced, and affinity-attenuated designs [30].

Calculation of intermolecular interactions between favipiravir-bound nsp12 designs

The intermolecular interactions between top-ranked affinity-attenuating and affinityenhancing favipiravir-nsp12 designs were identified using the Arpeggio web-server [31]. The total number of interactions obtained was the cumulative of the number of van der Waals interactions, hydrogen bonds, proximal contacts, polar contacts, hydrophobic contacts, aromatic contacts, and carbonyl interactions. The interaction figures were created using Schrödinger Maestro (Schrödinger Release 2016-4: Maestro, Schrödinger, New York).

\section{Results}

Analysis of nsp12-favipiravir interacting residues and selection of nsp12 residues for designing

The cryo-EM structure of SARS-CoV-2 RdRp in complex with favipiravir-RTP revealed the interacting residues between nsp12 and favipiravir (Figure 1a) [32]. A total of 72 residues of nsp12 were found to be interacting with favipiravir, of which those that interacted with the template:primer dsRNA were identified as essential for the catalytic activity of RdRp. Overall, 41 residues of nsp12 were identified and subjected to design; 
these were primarily located within $6 \AA$ distance of favipiravir. Certain critical residues, such as Asn691, Lys545, and Ser814, formed hydrogen bonds with favipiravir. Furthermore, favipiravir formed hydrogen bonds with $\mathrm{C} 10$, metal coordination of $\mathrm{Mg}^{2+}$, and a $\pi-\pi$ stacking with U20 of the template:primer dsRNA (Figure 1b, 1c).

\section{Identification of resistance mutations from MOE-based design experiments}

Next, we designed 41 shortlisted residues of nsp12 using resistance mutation scan methodology employed in Molecular Operating Environment (MOE), where each residue was mutated with only a single nucleotide polymorphism (SNP) of the wild-type sequence. As a result, the mutations were confined to SNPs to mimic the variations that may occur naturally during the evolution of the virus. Table 1 shows a list of SNPs sampled and designed for 41 favipiravir-interacting residues of nsp12. A total of 350 single point mutants potentially associated with the resistance development were generated, and the affinity between the designed proteins and favipiravir was calculated. The relative binding affinity of the mutation in comparison with the wild-type protein (dAffinity) was calculated. Here, a positive value indicates that the mutation had a lower affinity to favipiravir, suggesting that such mutant could become resistant to the ligand.

350 designs revealed that the dAffinity ranged from -0.54 to $0.52 \mathrm{kcal} / \mathrm{mol}$ (Supplementary Figure 1). Out of which, 152 designs retained positive dAffinity values, indicating they could reduce the affinity of nsp12 toward favipiravir. The dAffinity values of each of the 41 favipiravir-interacting residues with their corresponding single point resistant mutations are shown in Figures 2 and 3. Furthermore, a stringent dAffinity cutoff revealed that 13 mutants had dAffinities higher than $0.2 \mathrm{kcal} / \mathrm{mol}$ (Figure 4). These mutants with the highest dAffinity values can be significant for the development of the resistance against favipiravir. Notably, most of these 13 mutants originated from mutations in four nsp12 residues, namely His439, Cys622, Asp623, and Thr680 (Figure 4). Furthermore, certain residues, such as Asp452, Tyr456, Met542, Tyr546, Ala554, Trp617, Tyr619, Cys622, Arg624, Gly679, and Trp800 were susceptible to developing resistance during evolution if an immune and drug response was mounted on SARS-CoV2 , as revealed by their affinity profiles (Figures $2-4$ ). Using this alternative method, we 
then carried out a computational fitness test to sample only those mutations that were not lethal to the virus and that more likely to evolve naturally over time to assist $R d R p$ in maintaining structural and functional integrity.

\section{Rosetta-based design of favipiravir-binding nsp12 region and associated physicochemical features}

After we identified single point mutants that has the potential to induce favipiravir resistance, Rosetta-based ligand interface-design of the favipiravir-binding site of nsp12 in the nsp7-nsp8-nsp12-dsRNA complex was performed [24]. This was conducted to obtain the hotspot residues of nsp12 and better understand the mutational landscape during the emergence of drug resistance. Although 72 residues of nsp12 were found to interact with favipiravir, 41 were selected to design with backbone flexibility, as they did not interact with the template:primer dsRNA and were not involved in the catalytic activity. In this step, each of the 41 residues was mutated based on the corresponding SNPs to mimic the mutations that are more likely to occur naturally during the evolution of the protein (Table 1). During the design experiment, nsp12 residues other than the interfacial residues were only repacked. A total of 100,000 designs were generated, and their physicochemical parameters were analyzed. The 100,000 designs enclosing the favipiravir-binding site of nsp12 were categorized into affinity-enhancing and affinityattenuating designs based on their binding affinities and control values.

First, 100,000 designs were examined for Rosetta total scores versus their root mean square deviations (RMSDs). The Rosetta total score is the sum of individual energy terms, including physical forces and other statistical terms, whereas the RMSD is computed between the designed structures with respect to the wild-type nsp7-nsp8nsp12-template:primer dsRNA complex structure with favipiravir-RTP. It was observed that nearly all designs retained RMSDs below $1.5 \AA$, and more than half of them retained RMSDs below $1 \AA$, suggesting they did not considerably change from the initial complex structure during designing 41 interface residues of nsp12 and introducing mutations (Figure 5a). At this stage, we performed a control run, where 41 residues of nsp12 were only repacked without designing. This distinguished the affinity-attenuating and affinity- 
enhancing designs, indicating that the affinity-attenuating designs showed a minor increase in RMSDs in their overall structures and comparatively unfavorable energetics due to the introduction of missense mutations.

Second, the designs were analyzed for binding affinities between the designed nsp12 with favipiravir (represented as interface delta) versus the Rosetta total scores. The affinity-attenuating designs had considerably lower binding affinities and lower total scores than the affinity-enhancing and control designs (Figure 5b). This suggested that unfavorable energetics and lower total scores influenced the binding affinity in the affinityattenuating designs.

Third, we computed the RMSDs versus interface delta for the 100,000 designs. We found that several affinity-attenuating designs with lower binding affinities displayed higher RMSDs of over $1.2 \AA$, suggesting that the corresponding mutations destabilized the interactions (Figure $5 \mathrm{c}$ ). However, several affinity-attenuating designs with lower binding affinities retained RMSDs as compared to the affinity-enhancing and control designs as well, indicating that residue-specific side-chain movements could have contributed to the reduced binding affinities in those cases.

Finally, the interface delta of the designs was evaluated against the percentage sequence identities of the generated designs. Interestingly, although nsp12 has 41 residues interacting with favipiravir, only a few of them were potentially hotspot residues and prone to mutations (Figure $5 \mathrm{~d}$ ). On average, the designs retained a sequence identity of 97 to $98 \%$ as compared to the wild-type structure. The affinity-attenuating designs with lower binding affinities were less conserved than their counterparts (Figure 5d), demonstrating that nsp12 developed resistance against favipiravir with only selected mutations at a few hotspot residues whenever SARS-CoV-2 encounters evolutionary and immune/drug response.

\section{Mutational landscape and sequence variations in favipiravir-nsp12 binding designs}

The top 50 designs from each category (affinity-enhancing and affinity-attenuating designs) were further analyzed and compared to determine the key differences in the 
mutations and sequence diversities (Figure 6). Detailed analysis of the mutational landscape revealed that in both categories, residues His439, Asp452, Tyr456, Met542, Tyr546, Ala547, Ser549, Ala550, Lys551, Arg553, Ala554, Arg555, Thr556, Lys621, Cys622, Ala625, Arg631, Val662, Lys676, Gly679, Thr680, Ser681, Ala690, Ser692, Phe694, Asn695, Val763, Trp800, His810, His816, and Asp833 were identically sampled (Figure 6). However, certain wild-type residues, such as Trp617, Asp618, Tyr619, Asp623, and Ala762, exhibited the most diverse sequence variations between the two categories. Furthermore, few other residues, such as Gly616, Pro620, Arg624, Met626, and Lys798, showed mutations with a relatively lower number of sequence variations between the affinity-attenuating and affinity-enhancing designs (Figure 6). Therefore, a total of 10 residue sites exhibited sequence variations between the affinity-attenuating and affinity-enhancing designs. Notably, these residues that showed sequence variations and were highly susceptible to mutations belonged to the palm domain of RdRp. In addition, in the affinity-attenuating designs, residues at positions $617,618,619,623$, and 762 sampled a high number of extremely diverse amino acids (Figure 6).

A comparison of MOE-derived single point resistance-developing mutant with that of the Rosetta-generated mutational landscape demonstrated that both methods predicted certain commonly occurring mutants, such as D623A and D623G in affinityattenuating designs (Figures 2, 3, 4, and 6). Furthermore, the Rosetta-based design revealed a few more mutation types for hotspot residues. This sequence-specific conservation and diversity of the favipiravir-bound nsp12 designs indicated that, in the future, these hotspot residues could undergo selective mutations to establish a resistance to favipiravir and similar drugs. This can facilitate the spread and survival of SARS-CoV2 or a related virus.

\section{Validation of design protocols}

To confirm the predictive ability and accuracy of the MOE-based and Rosetta-based design methodologies, a well-defined remdesivir-resistant V557L mutant of SARS-CoV was assessed. The chikungunya virus and $\mathrm{H} 1 \mathrm{~N} 1$ influenza $A$ virus have been shown to develop resistance against favipiravir. However, since their 3D structures are not 
available, we validated our findings with SARS-CoV V557L mutant, as Val557 is conserved in the nsp12 of SARS-CoV-2. To evaluate whether our MOE and Rosetta design methodologies could rank L557 among the low-affinity designs, we scanned residues in the $557^{\text {th }}$ position with $A, D, E, G, I, L, M$, and $F$ residues and realized that V557L was ranked as the low-affinity mutant in the design calculations (Supplementary Figure 2). These observations validated the capability of our design methodologies to score and rank-order of the affinity-attenuating designs that can develop favipiravir resistance in SARS-CoV-2. However, because V557L is a remdesivir-resistant mutation of SARS-CoV, the SARS-CoV-2 may not mutate Val557 residue to acquire favipiravir resistance. Instead, it could use any other hotspot residues to develop resistance. Finally, mutations at the favipiravir binding site of $R d R p$ were obtained from the CoV-GLUE database, and their frequency of occurrence was determined. We observed that out of 134 mutations, 63 mutations were already predicted as resistant, as shown in our MOEbased design calculations, thus attaining $\sim 47 \%$ correlation match with the sequencing data (Figure 7). Notably, many high-frequency mutations such as A554S, A554T, A554V, W617C, Y619C, and H810Y were already found to be favipiravir resistant in our design calculations (Figure 7). However, it should be noted that the frequencies of the mutations recorded in the CoV-GLUE database may not directly correlate with the calculated dAffinities. As the pandemic advances and more sequencing data becomes available, it is possible that the other sampled mutations from our designs may evolve and correlate with the new sequences. In conclusion, the control experiments validated the design methodology in scoring and rank-ordering the affinity-attenuating designs, leading to the emergence of potential mutations for favipiravir resistance in SARS-CoV-2.

\section{Intermolecular interactions between favipiravir and nsp12 in the top-ranked affinity-attenuating and affinity-enhancing designs}

We examined the intermolecular interactions between favipiravir and nsp12 in the topscored affinity-attenuating and affinity-enhancing designs. The top-scored affinityenhancing design formed 237 interactions, while the affinity-attenuating design formed 221 interactions (Table 2). The van der Waals, proximal, and metal-complex interactions 
played a significant role in decreasing the affinity between favipiravir and nsp12 in the affinity-attenuating designs (Figures 8a, 8b). A ligand interaction diagram from the affinityattenuating and affinity-enhancing designs showed that the lack of a hydrogen bond between Asn691-favipiravir and metal-ion bonds significantly contributed to the lower binding affinity in the affinity-attenuating designs (Figures 8c, 8d).

\section{Discussion}

A combination of different strategies encompassing multiple vaccines and antiviral therapeutics is required for the effective and complete management of COVID-19. Recently, several vaccine candidates have received EUA for COVID-19 prevention in several countries. However, because RNA viruses mutate and recombine rapidly, preparing long-lasting vaccines and continually effective therapeutics is challenging, as evident from the dramatic shifts in vaccine use worldwide. The majority of vaccines in the pipeline focus on the S-protein. Recent data from several countries, such as the UK, South Africa, and Australia, demonstrate accumulating mutations in the S-protein, allowing the virus to escape immune recognition, thus rendering the vaccines less effective or even ineffective. This could jeopardize the success of approved COVID-19 vaccines as well as the chances of accomplishing herd immunity. In addition, a large population of the Western world ( $42 \%$ of Americans) is reluctant to receive vaccines. These findings suggest that a combination of different strategies encompassing multiple vaccines and antiviral therapeutics will be required to effectively manage COVID-19.

SARS-CoV-2 jumped to humans in late 2019 via a possible transmission through bats, civets, or pangolin. Therefore, SARS-CoV-2 may not be completely adapted to its human host due to its recent association with this host. However, since its emergence, the SARS-CoV-2 has undergone a considerable number of mutations and recombination events. Although the majority of mutations detected were neutral, these are gradually accumulating, leading to increased genomic diversity. Although the current rate of sequence variations among SARS-CoV-2 isolates is modest, this RNA virus has ample opportunity to generate multiple variants because of the rapid rate of mutations and recombination events over the course of the pandemic (https://www.gisaid.org/). All this 
increases the probability of the emergence of more infectious strains in the future [33]. According to the population genetics theory, the neutral and beneficial mutations that affect viral fitness can reach higher frequencies. The development and spread of a mutation are governed by several factors, including population growth, founder effects, range expansion, and random genetic drift, as well as by a potential positive selection pressure to confer enhanced transmissibility or drug and immune resistance. Therefore, SARS-CoV-2 may diverge into phenotypically distinct lineages as it establishes itself as an endemic human pathogen. The non-synonymous D614G mutation in the viral Sprotein was the first mutation detected in early March 2020 that rapidly dominated by July 2020 [34]. Although the D614G mutant isolate is not linked to increased mortality or clinical severity, it is more transmissible $[35,36]$ and enhances the viral replication rate $[37,38]$. This was followed by the discovery of several mutations in the S-protein RBD in mink and humans associated with mink farms [39]. By December 2020, a new lineage, B.1.1.7 (also called 501Y.V1), emerged in the UK and is spreading rapidly with a higher transmission rate [40]. This B.1.1.7 variant includes several mutations, of which eight mutations are in the S-protein [41]. Another lineage, B.1.351 (also called 501Y.V2), which is rapidly spreading in South Africa, has nine mutations in the S-protein. A third strain, P.1 (also called 501Y.V3), emerged in Brazil in December 2020. This P.1 variant contains a unique constellation of ten mutations, including three mutations in the spike protein receptor-binding domain: K417T, E484K, and N501Y. In March 2021, a highly transmissible variant, named B.1.617, that has around 15 mutations, emerged in India. Reports claim that this variant is responsible for the much severe second wave of infection in India. Because most of the current vaccines target the viral S-protein antigen, mutations in the S-protein can mediate virus escape from host antibodies and possibly weaken the efficacy of the vaccine. Therefore, multiple pharmacological options should be available for the complete and effective management of COVID-19.

Favipiravir and remdesivir are the key drugs currently used to treat patients with COVID-19 in several countries [42]. They are being tested in clinical trials globally for treating SARS-CoV-2 infection. Favipiravir is considerably cheaper than remdesivir and is, therefore, more affordable for developing and poorer countries. Like other drug therapies, a major challenge with the widespread use of remdesivir and favipiravir could 
be the potential development of resistance [21]. These drugs are nucleoside analogs and target the RdRp of SARS-CoV-2 to stop viral replication and transcription. The viral RdRp has a high mutation rate, which could facilitate antigenic drift in response to the host immune pressure and allow the emergence of resistance against the antivirals [43]. Although no natural favipiravir-resistant mutants have been found in influenza viruses [14, 44-48], an in vitro study has reported that the K229R mutation in the influenza virus RdRp induces favipiravir resistance [20]. Although the K229R mutation reduces the polymerase activity, the fitness cost of this mutation is compensated by a P653L mutation in RdRp [20]. Therefore, P653L mutation can restore RdRp activity while maintaining favipiravir resistance. A combination of $\mathrm{K} 229 \mathrm{R}$ and $\mathrm{P} 653 \mathrm{~L}$ mutations resulted in a virus strain that was 30 -fold less susceptible to favipiravir than the wild-type virus while maintaining the replication kinetics. Notably, significant favipiravir resistance was reported from experimental evolution studies in the enterovirus 71 [19] and Chikungunya virus [18]. Overall, these data indicate a potentially universal mechanism for favipiravir resistance functioning under evolutionary and survival pressure. The present study will help better understand the structural dynamics of susceptible hotspots and resistance mutations and their possible effects on the efficacy of favipiravir. The validation will require close monitoring of SARS-CoV-2 evolution using multiple high-throughput genome analyses and SNP evaluation in the coming days of the pandemic.

\section{Limitations of the study}

This study had a few limitations. First, computationally predicted resistance mutations of nsp12 against favipiravir should be validated using biochemical experiments to quantify the gain/loss and strength of interactions between nsp12 designs and favipiravir to get a complete understanding of the evolution of favipiravir-resistant mutations. Second, as the Rosetta-based interface design protocol aims to design mutants with improved binding affinity to favipiravir, a more appropriate design methodology and scoring function is required to scan for resistance mutations (designs that reduce binding affinity to a ligand). However, to ensure that our design methodology correctly predicted the affinityattenuating designs representing the resistance mutations, we conducted adequate 
control experiments on a SARS-CoV remdesivir-resistance mutant. Using the publicly available SARS-CoV-2 nsp12 sequences, we also ensured that the most plausible single point mutations characterizing the favipiravir resistance mutants are identified from our design experiments. In addition, the MOE-based resistance design approach was employed to ensure that the most plausible single point mutants characterizing the resistance mutations are identified and predicted quantitatively.

\section{Conclusions}

Pandemics have been affecting humanity for centuries. One positive aspect of the COVID-19 pandemic has been the unprecedented availability of scientific and technological advances that allowed the rapid development of therapeutic strategies. The SARS-CoV-2 has acquired only modest genetic variations to date with no specific mutation contributing to drug resistance. However, it is impossible to rule out the future development of resistance against the currently used drug regimens. Considering the critical importance of defining possible signatures for adaptation in SARS-CoV-2 against the current drugs, we conclude that our work will provide grounds for a better understanding of the favipiravir resistance and COVID-19 management and may offer structural strategies for the development of more effective therapeutics.

\section{ACKNOWLEDGEMENTS}

The authors are grateful to Dr. Kam Y.J. Zhang (Laboratory for Structural Bioinformatics, RIKEN, Yokohama) for his continuous support and valuable suggestions for improving the manuscript. The authors acknowledge RIKEN ACCC for the Hokusai supercomputing resources.

\section{AUTHOR CONTRIBUTIONS}

AKP carried out all the design experiments, data generation, and analysis. JD and VNU helped in analysis. AKP and TT conceived the study, participated in its design and 
coordination, and drafted the manuscript. All authors read and approved the final manuscript.

\section{CONFLICT OF INTEREST}

The authors declare no conflict of interest.

\section{References}

1 Gao, Y., Yan, L., Huang, Y., Liu, F., Zhao, Y., Cao, L., Wang, T., Sun, Q., Ming, Z., Zhang, L., Ge, J., Zheng, L., Zhang, Y., Wang, H., Zhu, Y., Zhu, C., Hu, T., Hua, T., Zhang, B., Yang, X., Li, J., Yang, H., Liu, Z., Xu, W., Guddat, L. W., Wang, Q., Lou, Z. and Rao, Z. (2020) Structure of the RNA-dependent RNA polymerase from COVID-19 virus. Science. 368, 779-782

2 Hillen, H. S., Kokic, G., Farnung, L., Dienemann, C., Tegunov, D. and Cramer, P. (2020) Structure of replicating SARS-CoV-2 polymerase. Nature. 584, 154-156

3 Yin, W., Mao, C., Luan, X., Shen, D. D., Shen, Q., Su, H., Wang, X., Zhou, F., Zhao, W., Gao, M., Chang, S., Xie, Y. C., Tian, G., Jiang, H. W., Tao, S. C., Shen, J., Jiang, Y., Jiang, H., Xu, Y., Zhang, S., Zhang, Y. and Xu, H. E. (2020) Structural basis for inhibition of the RNAdependent RNA polymerase from SARS-CoV-2 by remdesivir. Science. 368, 1499-1504

4 Wu, F., Zhao, S., Yu, B., Chen, Y. M., Wang, W., Song, Z. G., Hu, Y., Tao, Z. W., Tian, J. H., Pei, Y. Y., Yuan, M. L., Zhang, Y. L., Dai, F. H., Liu, Y., Wang, Q. M., Zheng, J. J., Xu, L., Holmes, E. C. and Zhang, Y. Z. (2020) A new coronavirus associated with human respiratory disease in China. Nature. 579, 265-269

5 Furuta, Y., Gowen, B. B., Takahashi, K., Shiraki, K., Smee, D. F. and Barnard, D. L. (2013) Favipiravir (T-705), a novel viral RNA polymerase inhibitor. Antiviral Res. 100, 446-454

6 Shiraki, K. and Daikoku, T. (2020) Favipiravir, an anti-influenza drug against lifethreatening RNA virus infections. Pharmacol Ther. 209, 107512

7 Chen, P. J., Chao, C. M. and Lai, C. C. (2020) Clinical efficacy and safety of favipiravir in the treatment of COVID-19 patients. J Infect

8 Sreekanth Reddy, O. and Lai, W. F. (2020) Tackling COVID-19 Using Remdesivir and Favipiravir as Therapeutic Options. Chembiochem

9 Misra, N. (2020) A short review on important drugs under clinical trial against Covid-19. Mini Rev Med Chem

10 Joshi, S., Parkar, J., Ansari, A., Vora, A., Talwar, D., Tiwaskar, M., Patil, S. and Barkate, H. (2021) Role of favipiravir in the treatment of COVID-19. International Journal of Infectious Diseases. 102, 501-508

11 Udwadia, Z. F., Singh, P., Barkate, H., Patil, S., Rangwala, S., Pendse, A., Kadam, J., Wu, W., Caracta, C. F. and Tandon, M. (2021) Efficacy and safety of favipiravir, an oral RNAdependent RNA polymerase inhibitor, in mild-to-moderate COVID-19: A randomized, comparative, open-label, multicenter, phase 3 clinical trial. Int J Infect Dis. 103, 62-71

12 Sangawa, H., Komeno, T., Nishikawa, H., Yoshida, A., Takahashi, K., Nomura, N. and Furuta, Y. (2013) Mechanism of action of T-705 ribosyl triphosphate against influenza virus RNA polymerase. Antimicrob Agents Chemother. 57, 5202-5208 
13 Jin, Z., Smith, L. K., Rajwanshi, V. K., Kim, B. and Deval, J. (2013) The ambiguous basepairing and high substrate efficiency of T-705 (Favipiravir) Ribofuranosyl 5'-triphosphate towards influenza A virus polymerase. PLoS One. 8, e68347

14 Baranovich, T., Wong, S. S., Armstrong, J., Marjuki, H., Webby, R. J., Webster, R. G. and Govorkova, E. A. (2013) T-705 (favipiravir) induces lethal mutagenesis in influenza A H1N1 viruses in vitro. J Virol. 87, 3741-3751

15 Naydenova, K., Muir, K. W., Wu, L.-F., Zhang, Z., Coscia, F., Peet, M. J., CastroHartmann, P., Qian, P., Sader, K., Dent, K., Kimanius, D., Sutherland, J. D., Löwe, J., Barford, D. and Russo, C. J. (2021) Structure of the SARS-CoV-2 RNA-dependent RNA polymerase in the presence of favipiravir-RTP. Proc Natl Acad Sci U.S.A. 118, e2021946118

16 van Dorp, L., Acman, M., Richard, D., Shaw, L. P., Ford, C. E., Ormond, L., Owen, C. J., Pang, J., Tan, C. C. S., Boshier, F. A. T., Ortiz, A. T. and Balloux, F. (2020) Emergence of genomic diversity and recurrent mutations in SARS-CoV-2. Infect Genet Evol. 83, 104351

17 Callaway, E. (2020) The coronavirus is mutating - does it matter? Nature. 585, 174-177

18 Delang, L., Segura Guerrero, N., Tas, A., Quérat, G., Pastorino, B., Froeyen, M., Dallmeier, K., Jochmans, D., Herdewijn, P., Bello, F., Snijder, E. J., de Lamballerie, X., Martina, B., Neyts, J., van Hemert, M. J. and Leyssen, P. (2014) Mutations in the chikungunya virus nonstructural proteins cause resistance to favipiravir (T-705), a broad-spectrum antiviral. J Antimicrob Chemother. 69, 2770-2784

19 Wang, Y., Li, G., Yuan, S., Gao, Q., Lan, K., Altmeyer, R. and Zou, G. (2016) In vitro assessment of combinations of enterovirus inhibitors against enterovirus 71. Antimicrob Agents Chemother. 60, 5357-5367

20 Goldhill, D. H., Te Velthuis, A. J. W., Fletcher, R. A., Langat, P., Zambon, M., Lackenby, A. and Barclay, W. S. (2018) The mechanism of resistance to favipiravir in influenza. Proc Natl Acad Sci U S A. 115, 11613-11618

21 Padhi, A. K., Shukla, R., Saudagar, P. and Tripathi, T. (2021) High-throughput rational design of the remdesivir binding site in the RdRp of SARS-CoV-2: implications for potential resistance. iScience. 24, 101992

22 Padhi, A. K. and Tripathi, T. (2021) Targeted design of drug binding sites in the main protease of SARS-CoV-2 reveals potential signatures of adaptation. Biochem Biophys Res Commun. 555, 147-153

23 Vilar, S., Cozza, G. and Moro, S. (2008) Medicinal chemistry and the molecular operating environment (MOE): application of QSAR and molecular docking to drug discovery. Curr Top Med Chem. 8, 1555-1572

24 Moretti, R., Bender, B. J., Allison, B. and Meiler, J. (2016) Rosetta and the Design of Ligand Binding Sites. Methods Mol Biol. 1414, 47-62

25 Alford, R. F., Leaver-Fay, A., Jeliazkov, J. R., O'Meara, M. J., DiMaio, F. P., Park, H., Shapovalov, M. V., Renfrew, P. D., Mulligan, V. K., Kappel, K., Labonte, J. W., Pacella, M. S., Bonneau, R., Bradley, P., Dunbrack, R. L., Jr., Das, R., Baker, D., Kuhlman, B., Kortemme, T. and Gray, J. J. (2017) The Rosetta All-Atom Energy Function for Macromolecular Modeling and Design. J Chem Theory Comput. 13, 3031-3048

26 Fleishman, S. J., Leaver-Fay, A., Corn, J. E., Strauch, E. M., Khare, S. D., Koga, N., Ashworth, J., Murphy, P., Richter, F., Lemmon, G., Meiler, J. and Baker, D. (2011) RosettaScripts: a scripting language interface to the Rosetta macromolecular modeling suite. PLoS One. 6, e20161

27 Kaufmann, K. W. and Meiler, J. (2012) Using RosettaLigand for small molecule docking into comparative models. PLoS One. 7, e50769

28 Sheahan, T. P., Sims, A. C., Zhou, S., Graham, R. L., Pruijssers, A. J., Agostini, M. L., Leist, S. R., Schäfer, A., Dinnon, K. H., 3rd, Stevens, L. J., Chappell, J. D., Lu, X., Hughes, T. M., George, A. S., Hill, C. S., Montgomery, S. A., Brown, A. J., Bluemling, G. R., Natchus, M. G., Saindane, M., Kolykhalov, A. A., Painter, G., Harcourt, J., Tamin, A., Thornburg, N. J., Swanstrom, 
R., Denison, M. R. and Baric, R. S. (2020) An orally bioavailable broad-spectrum antiviral inhibits SARS-CoV-2 in human airway epithelial cell cultures and multiple coronaviruses in mice. Sci Transl Med. 12, eabb5883

29 Singer, J., Gifford, R., Cotten, M. and Robertson, D. (2020) CoV-GLUE: A Web Application for Tracking SARS-CoV-2 Genomic Variation. Preprints

30 Crooks, G. E., Hon, G., Chandonia, J. M. and Brenner, S. E. (2004) WebLogo: a sequence logo generator. Genome Res. 14, 1188-1190

31 Jubb, H. C., Higueruelo, A. P., Ochoa-Montaño, B., Pitt, W. R., Ascher, D. B. and Blundell, T. L. (2017) Arpeggio: A Web Server for Calculating and Visualising Interatomic Interactions in Protein Structures. J Mol Biol. 429, 365-371

32 Naydenova, K., Muir, K. W., Wu, L.-F., Zhang, Z., Coscia, F., Peet, M. J., CastroHartmann, P., Qian, P., Sader, K., Dent, K., Kimanius, D., Sutherland, J. D., Löwe, J., Barford, D. and Russo, C. J. (2020) Structural basis for the inhibition of the SARS-CoV-2 RNA-dependent RNA polymerase by favipiravir-RTP. bioRxiv, 2020.2010.2021.347690

33 Padhi, A. K. and Tripathi, T. (2020) Can SARS-CoV-2 Accumulate Mutations in the SProtein to Increase Pathogenicity? ACS Pharmacol Transl Sci. 3, 1023-1026

34 Korber, B., Fischer, W. M., Gnanakaran, S., Yoon, H., Theiler, J., Abfalterer, W., Hengartner, N., Giorgi, E. E., Bhattacharya, T., Foley, B., Hastie, K. M., Parker, M. D., Partridge, D. G., Evans, C. M., Freeman, T. M., de Silva, T. I., McDanal, C., Perez, L. G., Tang, H., MoonWalker, A., Whelan, S. P., LaBranche, C. C., Saphire, E. O. and Montefiori, D. C. (2020) Tracking Changes in SARS-CoV-2 Spike: Evidence that D614G Increases Infectivity of the COVID-19 Virus. Cell. 182, 812-827.e819

35 Yurkovetskiy, L., Wang, X., Pascal, K. E., Tomkins-Tinch, C., Nyalile, T. P., Wang, Y., Baum, A., Diehl, W. E., Dauphin, A., Carbone, C., Veinotte, K., Egri, S. B., Schaffner, S. F., Lemieux, J. E., Munro, J. B., Rafique, A., Barve, A., Sabeti, P. C., Kyratsous, C. A., Dudkina, N. V., Shen, K. and Luban, J. (2020) Structural and Functional Analysis of the D614G SARS-CoV-2 Spike Protein Variant. Cell. 183, 739-751.e738

36 Volz, E., Hill, V., McCrone, J. T., Price, A., Jorgensen, D., O'Toole, Á., Southgate, J., Johnson, R., Jackson, B., Nascimento, F. F., Rey, S. M., Nicholls, S. M., Colquhoun, R. M., da Silva Filipe, A., Shepherd, J., Pascall, D. J., Shah, R., Jesudason, N., Li, K., Jarrett, R., Pacchiarini, N., Bull, M., Geidelberg, L., Siveroni, I., Goodfellow, I., Loman, N. J., Pybus, O. G., Robertson, D. L., Thomson, E. C., Rambaut, A. and Connor, T. R. (2020) Evaluating the Effects of SARS-CoV-2 Spike Mutation D614G on Transmissibility and Pathogenicity. Cell

37 Plante, J. A., Liu, Y., Liu, J., Xia, H., Johnson, B. A., Lokugamage, K. G., Zhang, X., Muruato, A. E., Zou, J., Fontes-Garfias, C. R., Mirchandani, D., Scharton, D., Bilello, J. P., Ku, Z., An, Z., Kalveram, B., Freiberg, A. N., Menachery, V. D., Xie, X., Plante, K. S., Weaver, S. C. and Shi, P. Y. (2020) Spike mutation D614G alters SARS-CoV-2 fitness. Nature

38 Hou, Y. J., Chiba, S., Halfmann, P., Ehre, C., Kuroda, M., Dinnon, K. H., 3rd, Leist, S. R., Schäfer, A., Nakajima, N., Takahashi, K., Lee, R. E., Mascenik, T. M., Graham, R., Edwards, C. E., Tse, L. V., Okuda, K., Markmann, A. J., Bartelt, L., de Silva, A., Margolis, D. M., Boucher, R. C., Randell, S. H., Suzuki, T., Gralinski, L. E., Kawaoka, Y. and Baric, R. S. (2020) SARS-CoV-2 D614G variant exhibits efficient replication ex vivo and transmission in vivo. Science. 370, 14641468

39 Oude Munnink, B. B., Sikkema, R. S., Nieuwenhuijse, D. F., Molenaar, R. J., Munger, E., Molenkamp, R., van der Spek, A., Tolsma, P., Rietveld, A., Brouwer, M., Bouwmeester-Vincken, N., Harders, F., Hakze-van der Honing, R., Wegdam-Blans, M. C. A., Bouwstra, R. J., GeurtsvanKessel, C., van der Eijk, A. A., Velkers, F. C., Smit, L. A. M., Stegeman, A., van der Poel, W. H. M. and Koopmans, M. P. G. (2020) Transmission of SARS-CoV-2 on mink farms between humans and mink and back to humans. Science

40 Volz, E., Mishra, S., Chand, M., Barrett, J. C., Johnson, R., Geidelberg, L., Hinsley, W. R., Laydon, D. J., Dabrera, G., O'Toole, Á., Amato, R., Ragonnet-Cronin, M., Harrison, I., Jackson, 
B., Ariani, C. V., Boyd, O., Loman, N. J., McCrone, J. T., Gonçalves, S., Jorgensen, D., Myers, R., Hill, V., Jackson, D. K., Gaythorpe, K., Groves, N., Sillitoe, J., Kwiatkowski, D. P., Flaxman, S., Ratmann, O., Bhatt, S., Hopkins, S., Gandy, A., Rambaut, A. and Ferguson, N. M. (2021) Transmission of SARS-CoV-2 Lineage B.1.1.7 in England: Insights from linking epidemiological and genetic data. medRxiv, 2020.2012.2030.20249034

41 Rambaut, A., Loman, N., Pybus, O., Barclay, W., Barrett, J., Carabelli, A., Connor, T., Peacock, T., Robertson, D. L. and Volz, E. (2020) Preliminary genomic characterisation of an emergent SARS-CoV-2 lineage in the UK defined by a novel set of spike mutations. ed. $)^{\wedge}$ eds.)

42 Mishra, S. K. and Tripathi, T. (2020) One year update on the COVID-19 pandemic: Where are we now? Acta Trop. 214, 105778

43 Pauly, M. D., Procario, M. C. and Lauring, A. S. (2017) A novel twelve class fluctuation test reveals higher than expected mutation rates for influenza $A$ viruses. Elife. 6

44 Furuta, Y., Takahashi, K., Fukuda, Y., Kuno, M., Kamiyama, T., Kozaki, K., Nomura, N., Egawa, H., Minami, S., Watanabe, Y., Narita, H. and Shiraki, K. (2002) In vitro and in vivo activities of anti-influenza virus compound T-705. Antimicrob Agents Chemother. 46, 977-981

45 Daikoku, T., Yoshida, Y., Okuda, T. and Shiraki, K. (2014) Characterization of susceptibility variants of influenza virus grown in the presence of T-705. J Pharmacol Sci. 126, 281-284

46 Bank, C., Renzette, N., Liu, P., Matuszewski, S., Shim, H., Foll, M., Bolon, D. N., Zeldovich, K. B., Kowalik, T. F., Finberg, R. W., Wang, J. P. and Jensen, J. D. (2016) An experimental evaluation of drug-induced mutational meltdown as an antiviral treatment strategy. Evolution. 70, 2470-2484

47 Marathe, B. M., Wong, S. S., Vogel, P., Garcia-Alcalde, F., Webster, R. G., Webby, R. J., Najera, I. and Govorkova, E. A. (2016) Combinations of Oseltamivir and T-705 Extend the Treatment Window for Highly Pathogenic Influenza A(H5N1) Virus Infection in Mice. Sci Rep. 6, 26742

48 Takashita, E., Ejima, M., Ogawa, R., Fujisaki, S., Neumann, G., Furuta, Y., Kawaoka, Y., Tashiro, M. and Odagiri, T. (2016) Antiviral susceptibility of influenza viruses isolated from patients pre- and post-administration of favipiravir. Antiviral Res. 132, 170-177 


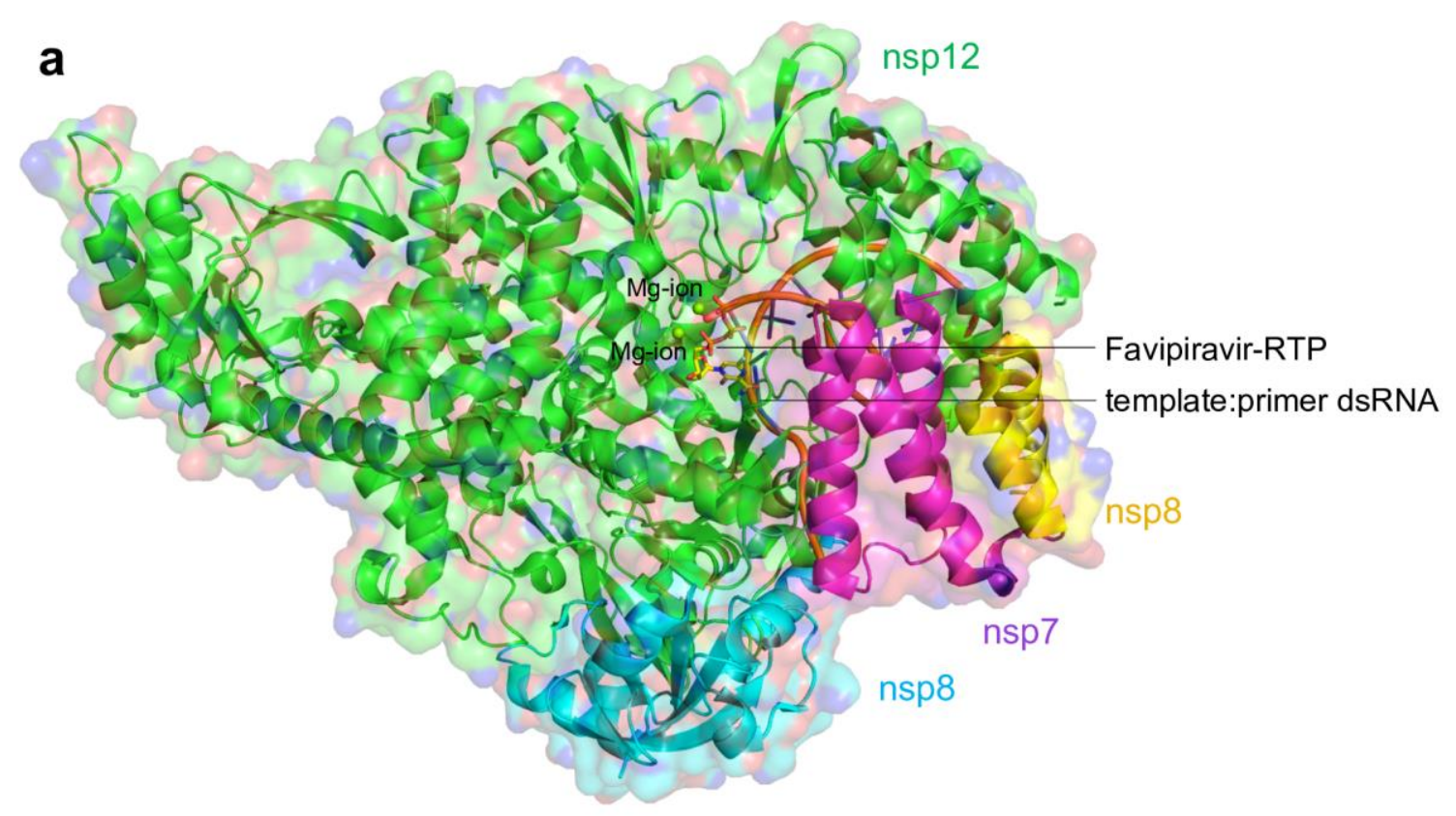

b

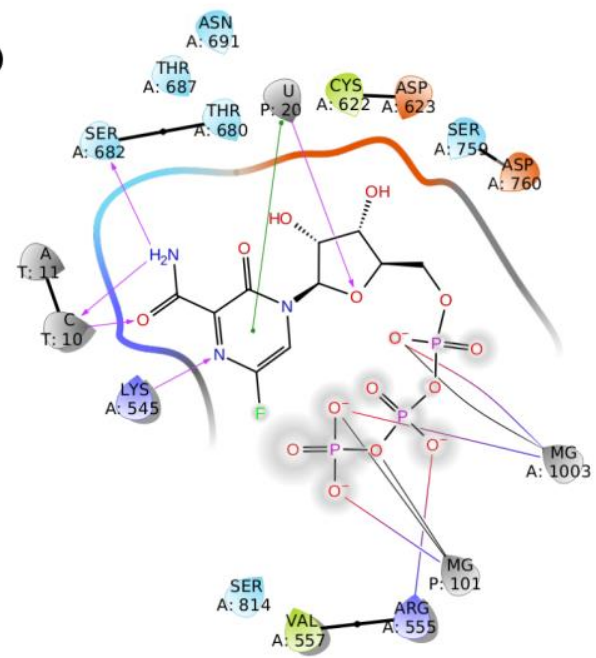

C

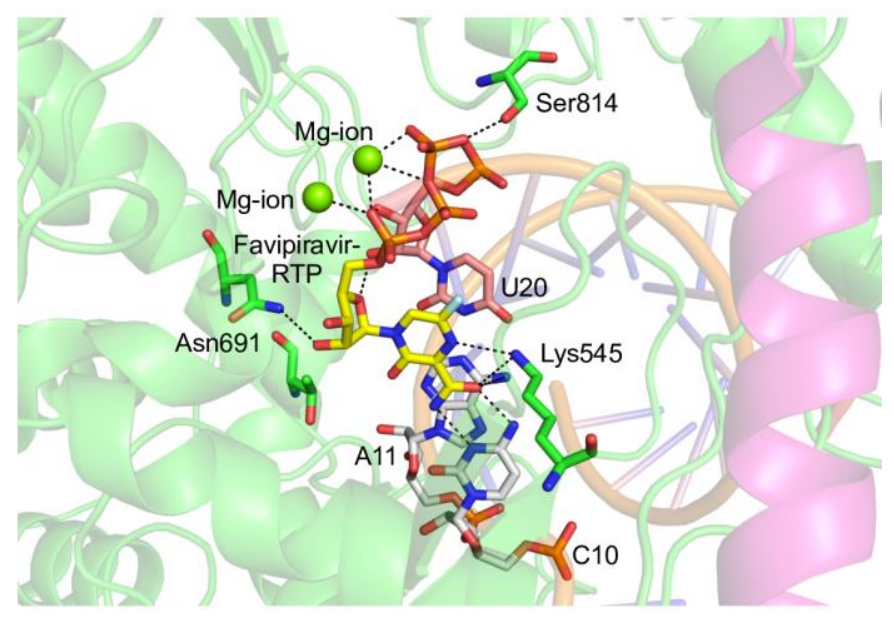

Charged (negative
Charged (positive)
Glycine
Hydrophobic
Metal

Polar
Unspecified residue
Water
Hydration site
$\times \quad$ Hydration site (displaced)

$\ldots$ Distance
$\Rightarrow$ H-bond
$=$ Metal coordination
$\because$ Pi-Pi stacking
$\rightarrow$ Pi-cation

- Salt bridge

Solvent exposure

Figure 1. Structure of the SARS-CoV-2 RdRp complex. (a) The cryo-EM structure of SARS-CoV-2 RdRp in complex with the template:primer dsRNA and favipiravir-RTP. The nsp7, nsp8, and nsp12 domains are labeled with respective colors, and catalytic $\mathrm{Mg}^{2+}$ ion is shown. The template:primer dsRNA and favipiravir-RTP are shown as a cartoon and stick model, respectively. (b) Ligand interaction diagram of favipiravir-RTP bound to the template:primer dsRNA. (c) Intermolecular interactions and interacting residues of nsp12 and RNA bases of favipiravir-RTP (yellow) and $\mathrm{Mg}^{2+}$ (green sphere) coordinating the favipiravir-RTP. Hydrogen bonds are shown as black dashes. 


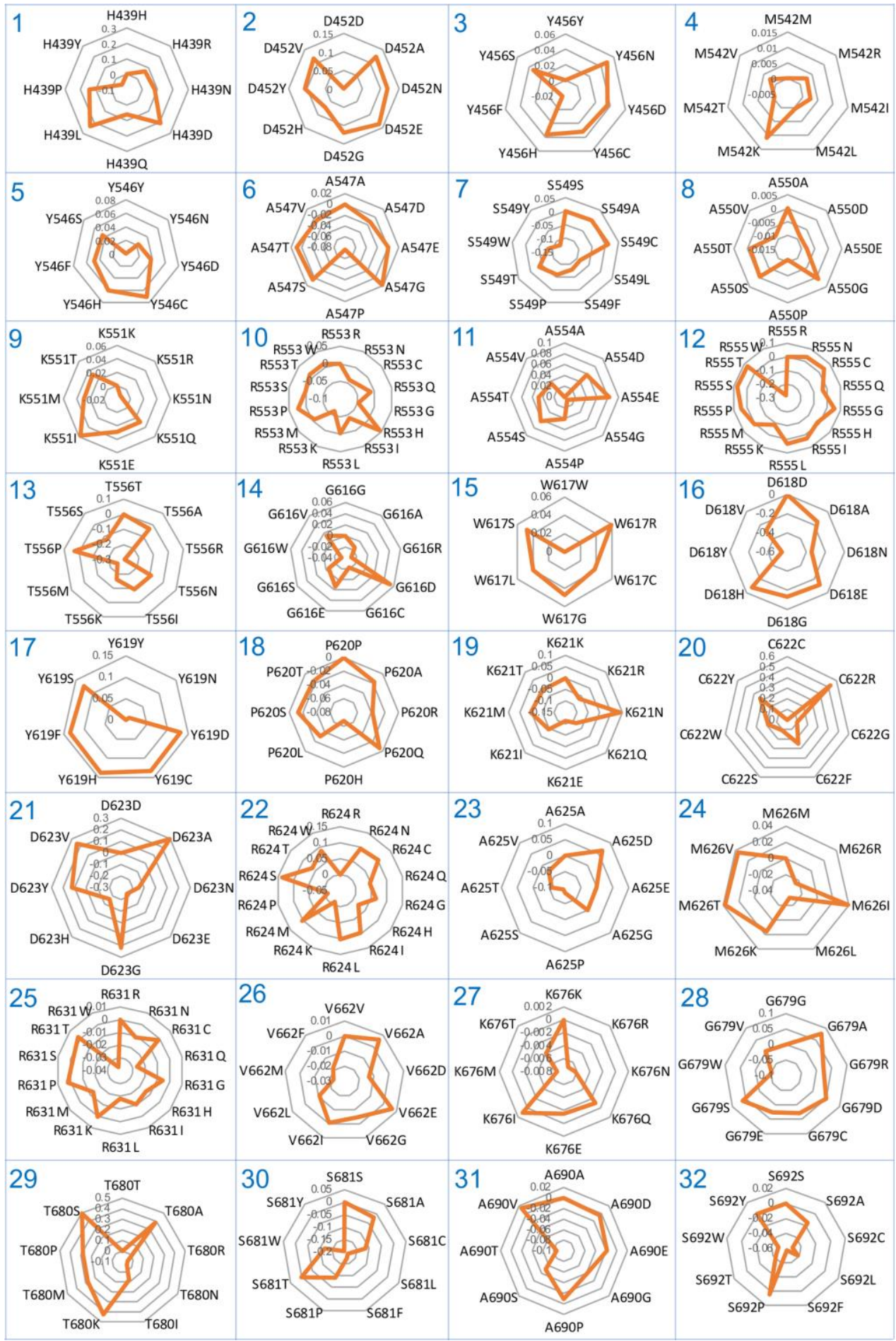

Figure 2. Relative binding affinities of designs from the favipiravir-interacting nsp12 residues. Radar plots showing the MOE-derived relative binding affinities (dAffinities) for favipiravir-interacting nsp12 residues and their single point mutant designs. The radar plots for (1-32) interacting residues and their corresponding mutated designs are labeled, and dAffinity values are highlighted. 


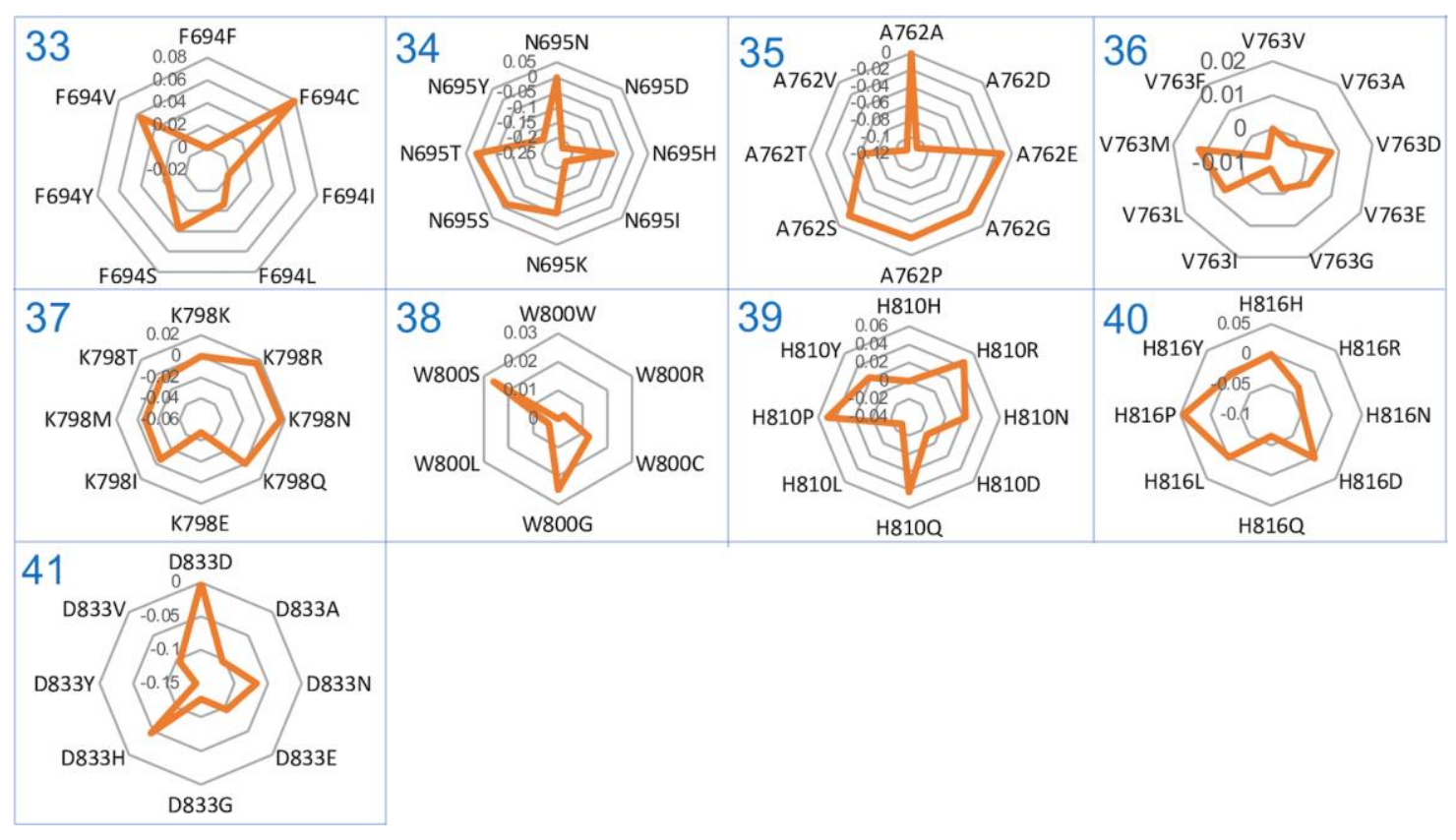

Figure 3. Relative binding affinities of designs from favipiravir-interacting nsp12 residues. The radar plots for (33-41) interacting residues. 


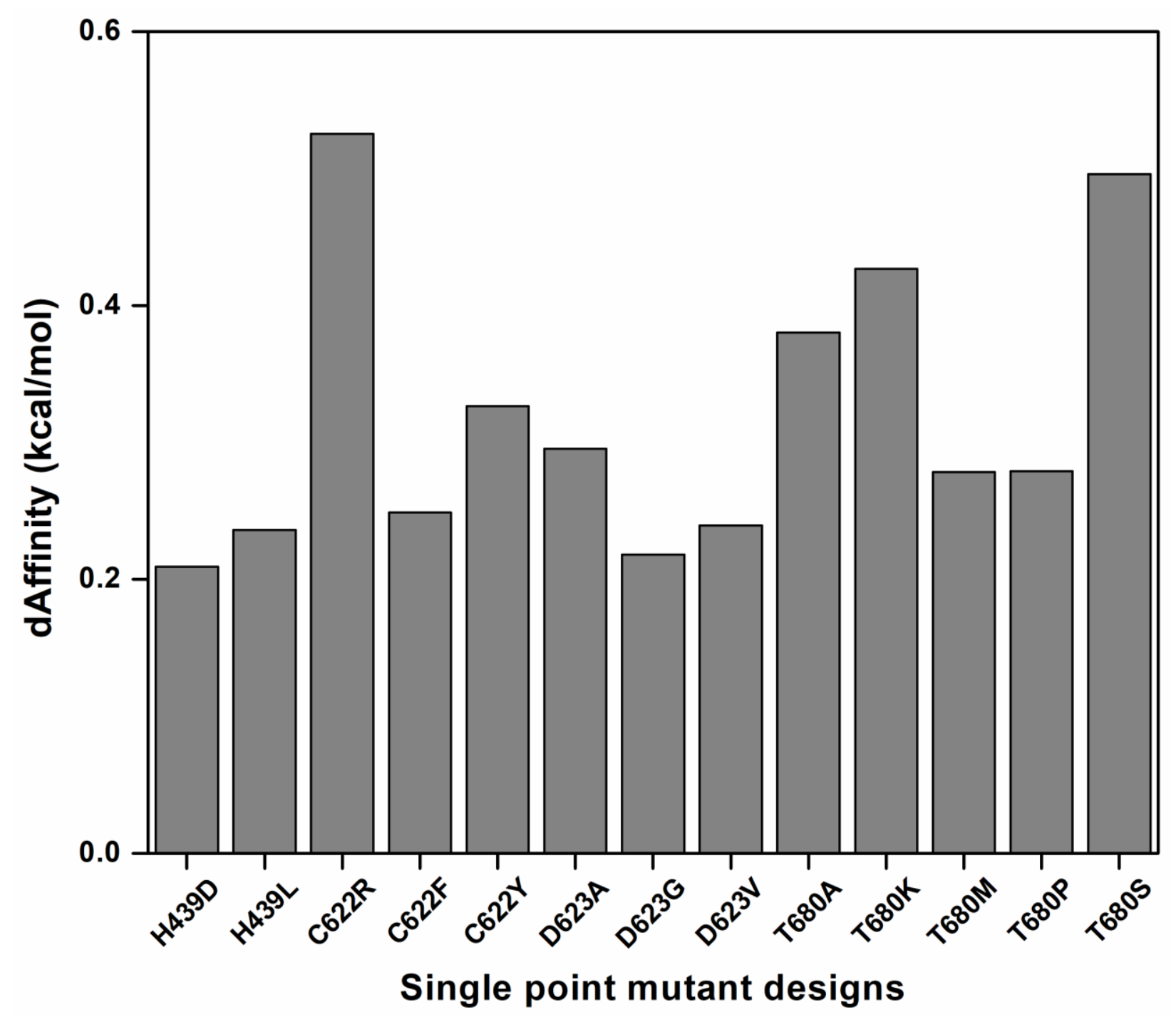

Figure 4. Relative binding affinities of the most plausible designs for favipiravir resistance. Bar plot showing the dAffinities for the most plausible favipiravir-interacting nsp12 residues and their single point mutant designs. These designs exhibited dAffinities higher than $0.2 \mathrm{kcal} / \mathrm{mol}$, suggesting potential resistance to favipiravir. 


\section{a}

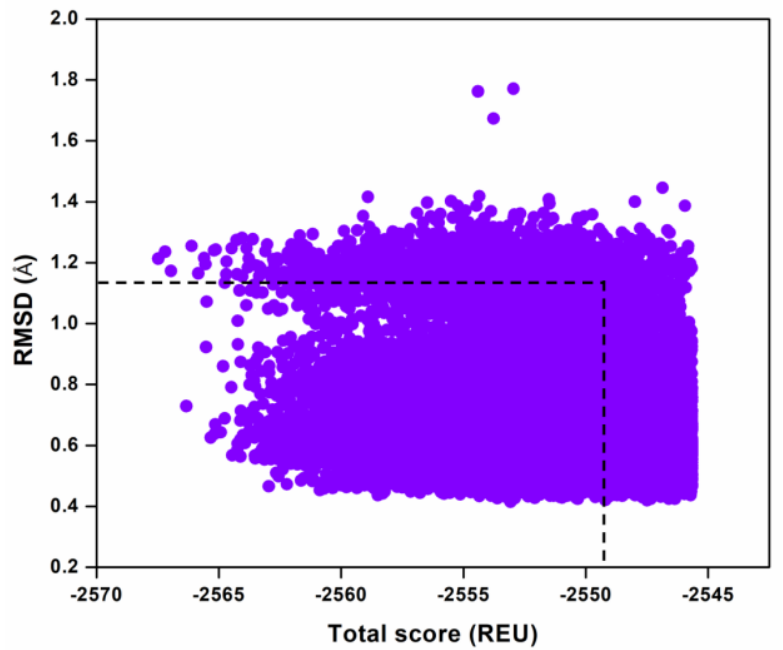

C

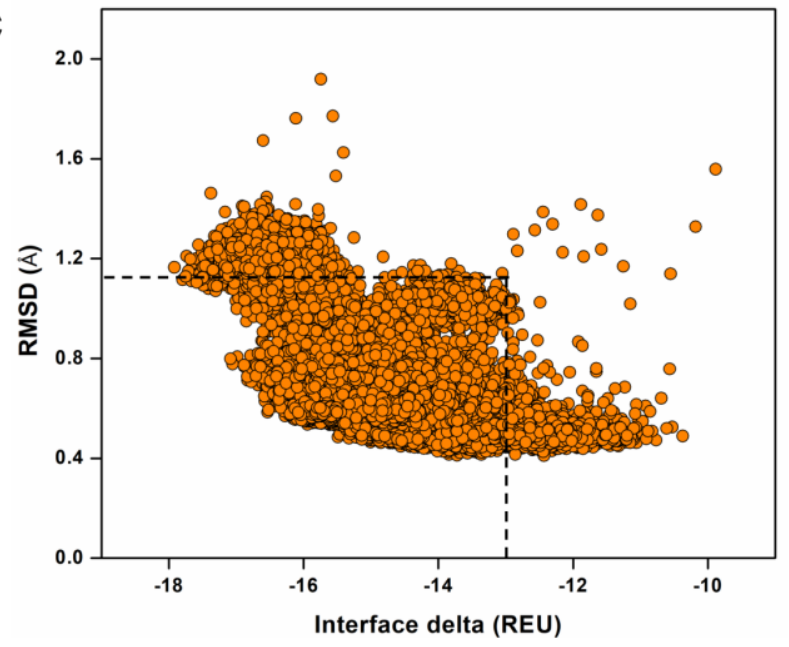

b

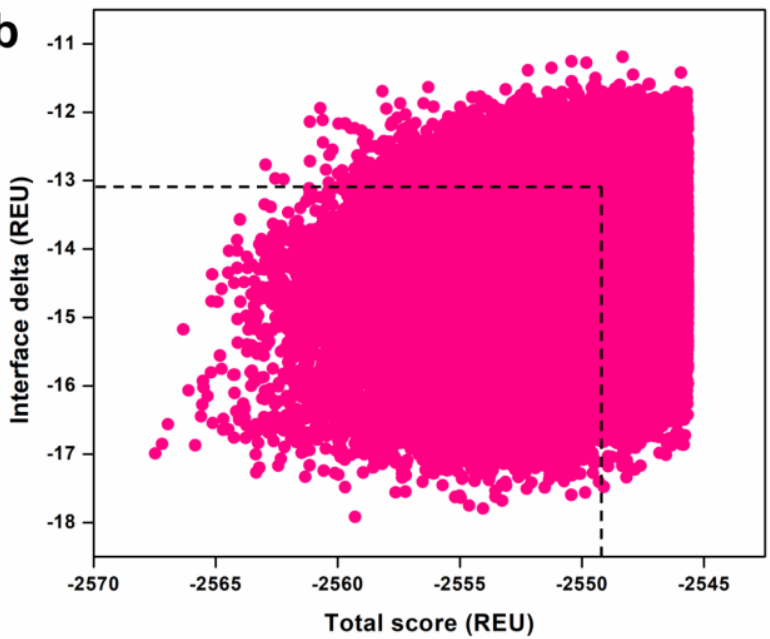

d

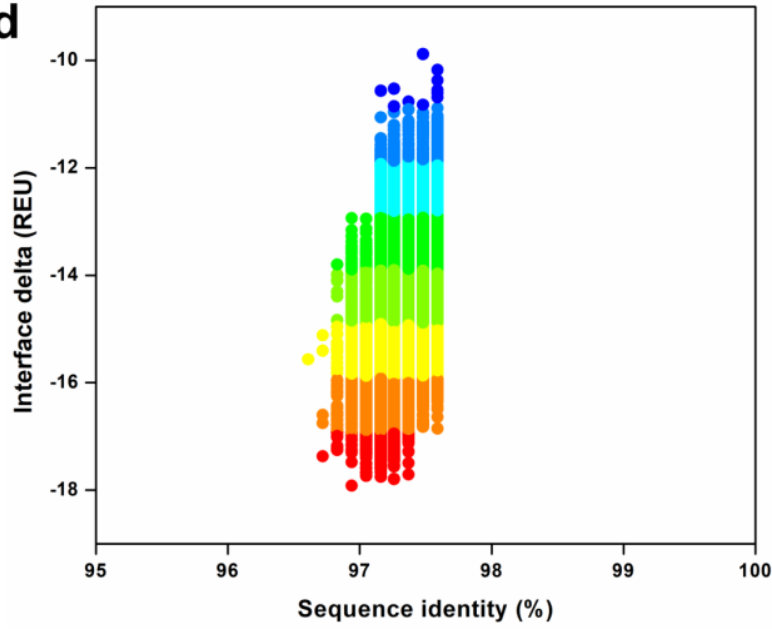

Figure 5. Structural and physicochemical parameters from the ligand-based Rosetta interface design of the favipiravir-RdRp interacting complex. (a) Rosetta total score vs. RMSD of 100,000 designs of the favipiravir-bound RdRp complex. (b) Rosetta total score vs. interface delta (binding affinity) of the designs between favipiravir and the RdRp complex. (c) Interface delta vs. RMSD of the designs. (d) Interface delta vs. percent sequence identity of favipiravir-interacting residues of the RdRp complex shows the distribution of designs. Blue to red color indicates designs with low to high binding affinities between favipiravir and RdRp complex. In panels $(a-c)$, the dashed boxes represent the control values, where favipiravir-interacting residues were only repacked and not designed. 


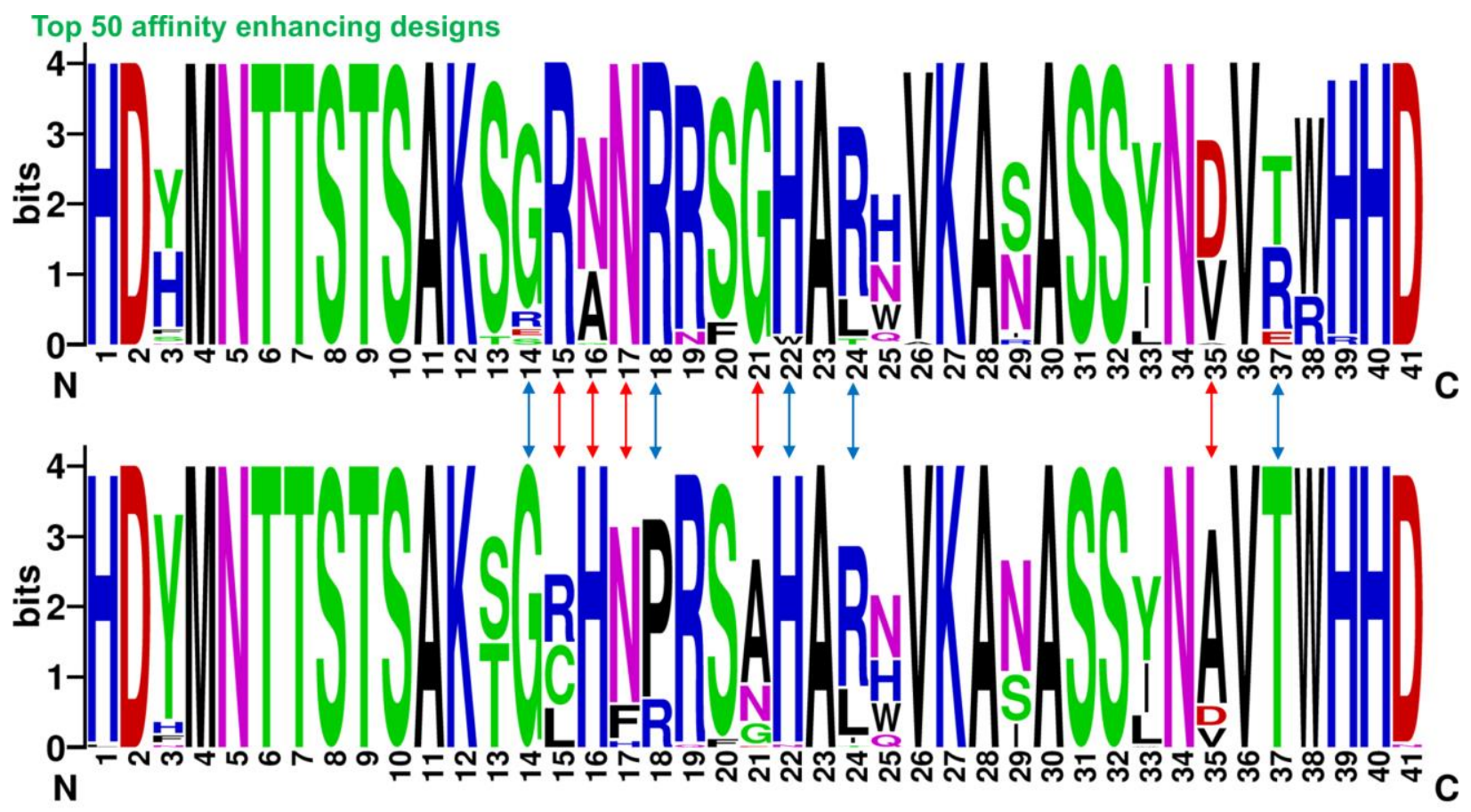

Top 50 affinity attenuating designs

(1) His439, (2) Asp452, (3) Tyr456, (4) Met542, (5) Tyr546, (6) Ala547, (7) Ser549, (8) Ala550, (9) Lys551, (10) Arg553, (11) Ala554, (12) Arg555, (13) Thr556, (14) Gly616, (15) Trp617, (16) Asp618, (17) Tyr619, (18) Pro620, (19) Lys621, (20) Cys622, (21) Asp623, (22) Arg624, (23) Ala625, (24) Met626, (25) Arg631, (26) Val662, (27) Lys676, (28) Gly679, (29) Thr680, (30) Ser681, (31) Ala690, (32) Ser692, (33) Phe694, (34) Asn695, (35) Ala762, (36) Val763, (37) Lys798, (38) Trp800, (39) His810, (40) His816, (41) Asp833

Figure 6. Sequence logos showing the type and frequency of designed favipiravirnsp12 interacting residues. Sequence logos of the 50 top-scored affinity-enhancing vs. affinity-attenuating designs are displayed. The $\mathrm{X}$-axis represents the residue index, and the $\mathrm{Y}$-axis shows the sequence conservation of an amino acid at that position. The height of symbols within the stack indicates the relative frequency of a particular amino acid at that position. The red arrow shows residues that experienced diverse sequence variations between the two groups with a relatively higher number of sampled amino acids, whereas the blue arrow shows residues that exhibited diverse mutations between the two groups but with fewer amino acids sampled. 


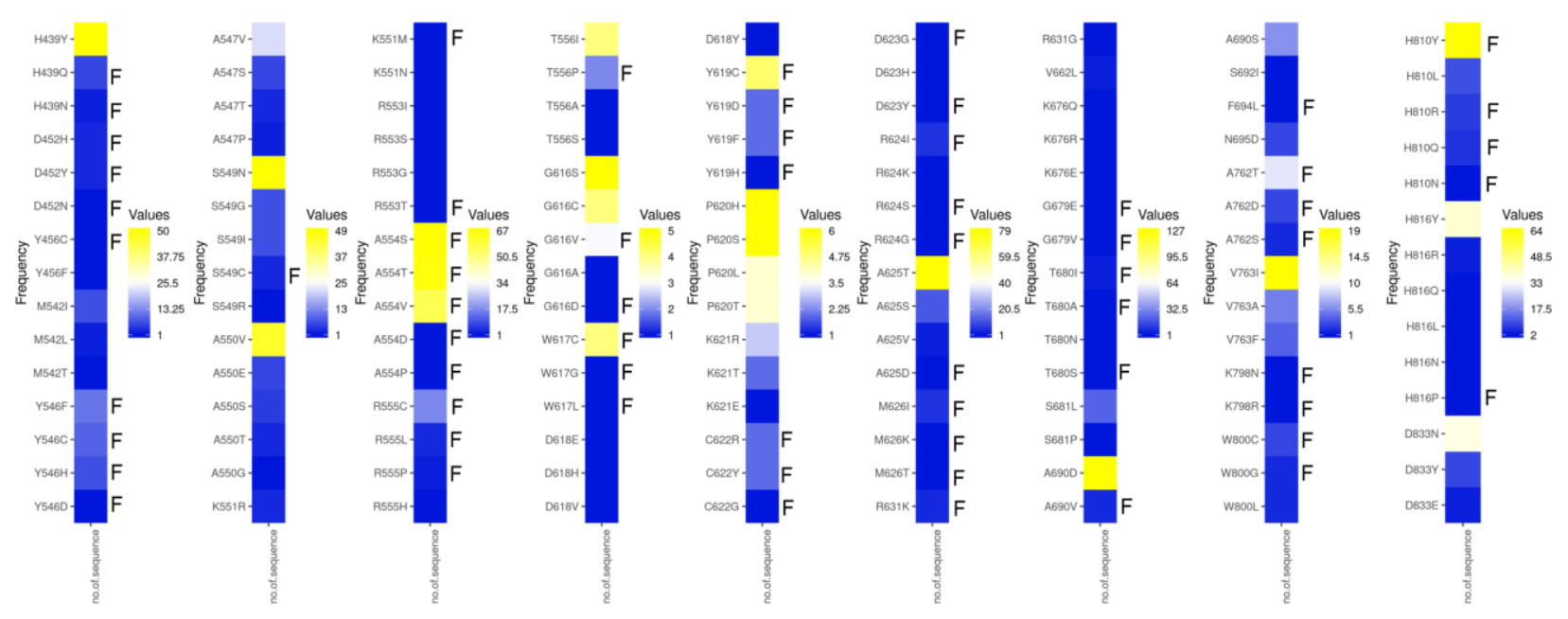

Figure 7. Heatmaps showing the mutations and their frequencies in the favipiravir binding site of nsp12 obtained from the CoV-GLUE database. Mutations in the favipiravir binding site of SARS-CoV-2 nsp12 obtained from the CoV-GLUE database are shown. The frequencies of the mutations among the COVID-19 cases ranging from lower to higher numbers are denoted from blue to yellow. The computed mutants that developed resistance towards favipiravir are marked as ' $F$ ' adjacent to the mutants. Out of 134 mutations, 63 were predicted as resistant and selected from our design computations, thus attaining $\sim 47 \%$ correlation and match with the sequencing data. 

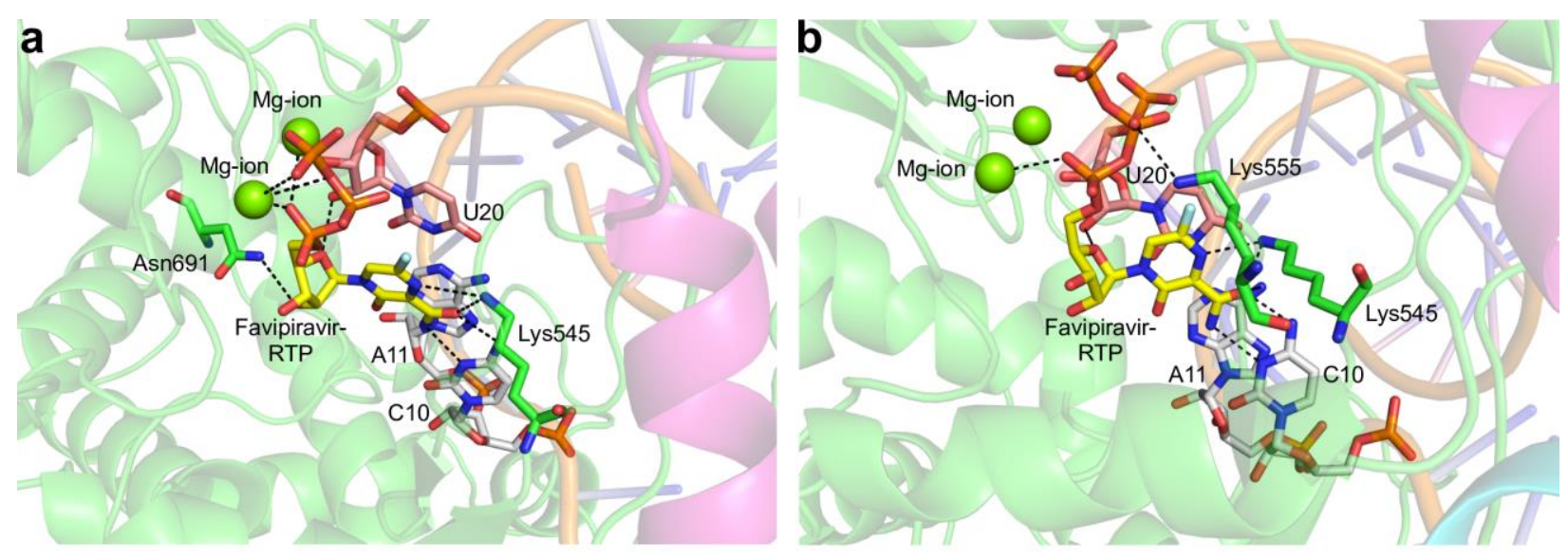

C

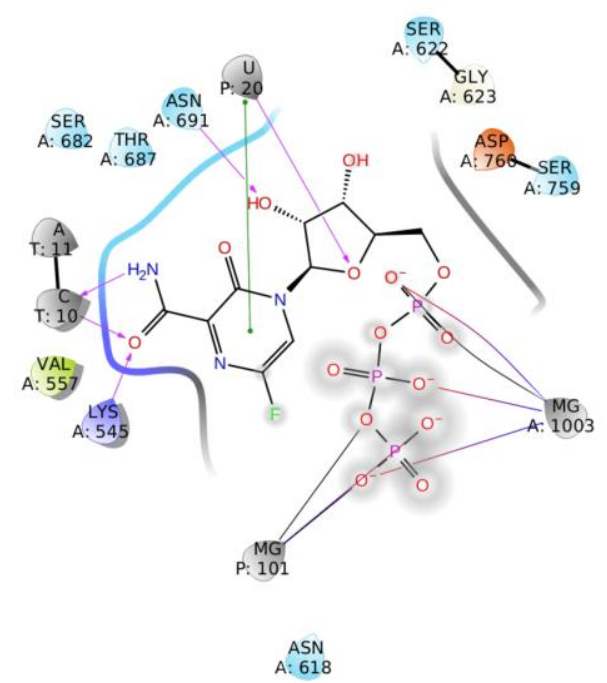

d

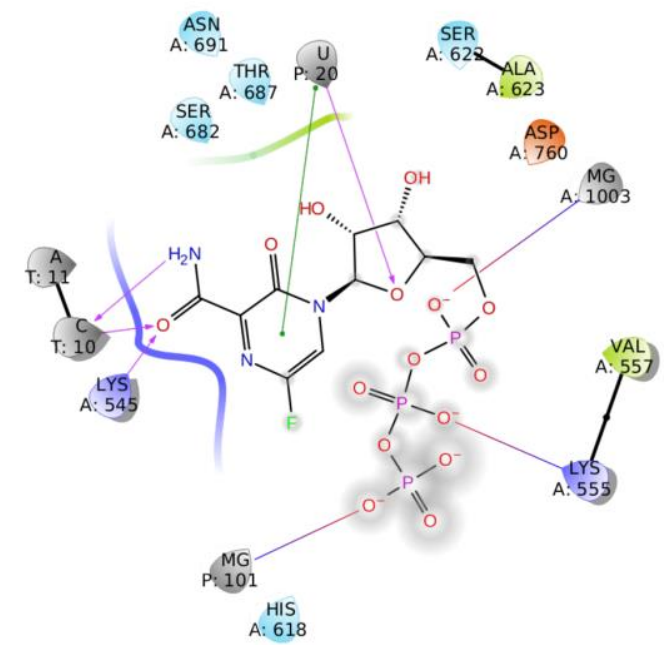

$\begin{array}{lll}\text { Charged (negative) } & \begin{array}{l}\text { Polar } \\ \text { Unspecified residue }\end{array} & \rightarrow \text { Distance } \\ \text { Charged (positive) } & \text { Water } & - \text { Hend } \\ \text { Glycine } & \text { Hydration site } & - \text { Mi-Pi stacking } \\ \text { Hydrophobic } & \text { Hydration site (displaced) } & \longrightarrow \text { Pi-cation } \\ \text { Metal } & \end{array}$

- Salt bridge

Solvent exposure

Figure 8. Intermolecular interactions and ligand interaction diagrams between favipiravir and the RdRp complex for the top-scoring designs. Intermolecular interactions between favipiravir and the RdRp complex for the top-scored (a) affinityenhancing designs and (b) affinity-attenuating designs. Interacting residues of corresponding nsp12 (green) and RNA bases with favipiravir (yellow) and $\mathrm{Mg}^{2+}$ (green sphere) coordinating the favipiravir-RTP are shown. Hydrogen bonds are shown as black dashes. (c) 2D ligand interaction diagrams between favipiravir and the RdRp complex for the top-scored affinity-enhancing and (d) affinity-attenuating design. The intermolecular interactions are labelled in the legend. 
Table 1. Favipiravir-interacting nsp12 residues that are designed with corresponding SNPs of the wild-type sequence to emulate the mutations that are more likely to happen naturally over the evolution of the protein.

\begin{tabular}{|c|c|c|c|c|c|}
\hline S. No & $\begin{array}{l}\text { Nsp12-favipiravir } \\
\text { interacting and } \\
\text { designed wild-type } \\
\text { residues }\end{array}$ & $\begin{array}{l}\text { Sampled SNPs in } \\
\text { designs }\end{array}$ & S. No & $\begin{array}{l}\text { Nsp12-favipiravir } \\
\text { interacting and } \\
\text { designed wild-type } \\
\text { residues }\end{array}$ & $\begin{array}{l}\text { Sampled SNPs in } \\
\text { designs }\end{array}$ \\
\hline 1 & His439 & RNDQLPY & 22 & Arg624 & NCQGHILKMPSTW \\
\hline 2 & Asp452 & ANEGHYV & 23 & Ala625 & DEGPSTV \\
\hline 3 & Tyr456 & NDCHFS & 24 & Met626 & RILKTV \\
\hline 4 & Met542 & RILKTV & 25 & Arg631 & NCQGHILKMPSTW \\
\hline 5 & Tyr546 & NDCHFS & 26 & Val662 & ADEGILMF \\
\hline 6 & Ala547 & DEGPSTV & 27 & Lys676 & RNQEIMT \\
\hline 7 & Ser549 & ACLFPTWY & 28 & Gly679 & ARDCESWV \\
\hline 8 & Ala550 & DEGPSTV & 29 & Thr680 & ARNIKMPS \\
\hline
\end{tabular}




\begin{tabular}{|c|c|c|c|c|c|}
\hline 9 & Lys551 & RNQEIMT & 30 & Ser681 & ACLFPTWY \\
\hline 10 & Arg553 & NCQGHILKMPSTW & 31 & Ala690 & DEGPSTV \\
\hline 11 & Ala554 & DEGPSTV & 32 & Ser692 & ACLFPTWY \\
\hline 12 & Arg555 & NCQGHILKMPSTW & 33 & Phe694 & CILSYV \\
\hline 13 & Thr556 & ARNIKMPS & 34 & Asn695 & DHIKSTY \\
\hline 14 & Gly616 & ARDCESWV & 35 & Ala762 & DEGPSTV \\
\hline 15 & Trp617 & RCGLS & 36 & Val763 & ADEGILMF \\
\hline 16 & Asp618 & ANEGHYV & 37 & Lys798 & RNQEIMT \\
\hline 17 & Tyr619 & NDCHFS & 38 & Trp800 & RCGLS \\
\hline 18 & Pro620 & ARQHLST & 39 & His810 & RNDQLPY \\
\hline 19 & Lys621 & RNQEIMT & 40 & His816 & RNDQLPY \\
\hline 20 & Cys622 & RGFSWY & 41 & Asp833 & ANEGHYV \\
\hline 21 & Asp623 & ANEGHYV & & & \\
\hline
\end{tabular}


Table 2. Detailed intermolecular interactions formed between favipiravir and nsp12 binding residues in the two top-scoring affinity-enhancing and affinity-attenuating designs.

\begin{tabular}{|l|l|l|l|l|}
\hline & \multicolumn{4}{|c|}{ Favipiravir-nsp12 } \\
\hline Types of interactions & $\begin{array}{c}\text { Affinity- } \\
\text { enhancing } \\
\text { design1 }\end{array}$ & $\begin{array}{c}\text { Affinity- } \\
\text { attenuating } \\
\text { design1 }\end{array}$ & $\begin{array}{c}\text { Affinity- } \\
\text { enhancing } \\
\text { design2 }\end{array}$ & $\begin{array}{c}\text { Affinity- } \\
\text { attenuating } \\
\text { design2 }\end{array}$ \\
\hline Van der Waals interactions & 3 & 1 & 2 & 2 \\
\hline Proximal interactions & 217 & 196 & 227 & 217 \\
\hline Polar contacts & 8 & 8 & 7 & 8 \\
\hline Hydrogen bonds & 5 & 6 & 5 & 5 \\
\hline Ionic interactions & 2 & 10 & 2 & 10 \\
\hline Metal complex interactions & 2 & 0 & 2 & 0 \\
\hline $\begin{array}{l}\text { Total number of } \\
\text { interactions }\end{array}$ & 237 & $\mathbf{2 2 1}$ & $\mathbf{2 4 5}$ & $\mathbf{2 4 2}$ \\
\hline
\end{tabular}

